\title{
SALE PRICES OF INCUNABULA,
} 1904.

\author{
GERMANY.
}

Mainz.

Schöffer with Fust. 1462. Biblia Latina. P. 79. [1343.] 2 ff onb. f. 2s. $6 d$.

Schöffer. 1472. Biblia Latina. P. 98. [1344.] $£ 4$

Schöffer. 1474. Joh. de Turrecremata: Expositio psalterii. P. 105. [1752.] $€ 5$ ros.

Reuwich. 1486. Bernard von Breydenbach: Reise ins heilige Land. P. 157. [1347.] imp. $\ell_{4} 4$ s.

Meydenbach. 1491. Hortus sanitatis. P. 160. [2434] imp. $£ 3$ Ios. [1875.] £45.

\section{Strassburg.}

Mentelin. n.d. Aristotelis ethica, etc. P. 224. [1338.] f10 10s. R. printer. n.d. Dionysius de Burgo: Comment. super Valerium Maximum. P. 237. [1361.] $£^{6}$ 1os.

Eggestein. n.d. Adrianus Carthusiensis: De remediis utriusque fortunae. P. 275. [1334.] \&5.

Eggestein. n.d. Johannes Damascenus: Gesta Barlanem et Josaphat. P. 290. [4157.] f4.

Schott. 1483. Otto von Passau: Die 24 Alten. Now P. 391 A.

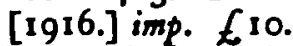

Schott. n.d. Leon. de Utino: Sermones de sanctis. P. 394- [1409.] fil ros.

Reinhard of Grüningen. 1497. Baldung: Aphorismi. P. 477. [1795.] f3 5 s.

Reinhard of Grüningen. 1497. Locher: Paneggrici ad Maximilianum: etc. P. 483. [1658.] 633 s.

Reinhard of Graningen. 1499. Terentius: Comoediae. P. 488. [1951.] f6 15 s.

Reinhard of Graningen. 1500. Hier. Braunschweig: Distillirbuch. P. 493. [4636.] $f 4$.

Reinhard of Grüningen. n.d. Nicolaus Panormitanus: Processus 12 judiciarius. P. 499 or 500 . [1 381.$]+\ell_{18} 5 s$. 
Kơn.

Zel. n.d. Augustinus: Enchiridion de fide. P. 810. [1341.] fel. n n.d. Aeneas Sylvius: Epistula ad Mahumetem. P. 8I6. [1335.] £3 3s.

ter Hoernen. 1474. Albertus Magnus: Sermones de tempore et sanctis. P. +936. [1336.] £3 3s.

Printer of Dares. Liber Alexandri de praeliis. Not in P. H. 778. [1785.] 64 .

Koelhoff. 1487. Johannes Chrysostomus: Sermones de patientia Job, etc. P. 1070. [2405.] $\& 36$ s.

Printer of Augustinus de Fide. n.d. Gerson: De laude scriptorum. P. 1097. [1 $3^{81}$. $]$ \& $f_{1} 85^{5}$.

Winters. 1476. Rolewinck: Fasciculus temporum. P. +1160. [1364.] \&4 ros.

Winters, n.d. Nider: de contractibus mercatorum. P. +1180. [1381.] $\neq £ 185 s$.

Quentell. 1479. Astesanus: Summa. P. 1236. [4261.] imp. $€ 5$. [3518.] Eio.

Quentell. 1479. Rolewinck: Fasciculus temporum. P. 1240. [1854.] $£ 33^{\text {s. }}$

Koelhoff. 1499. Cronica van Coellen. P. 1464. [1 569.] EI 7 $15 s$. [1834.] Mended and wormed E3 35. [1359.] f 15 los.

Cornelis, of Zierikeee. n.d. Nicodemus: Evangelium. P. 1507. [1395.] f3.

Cornelis, of Zierikzee. n.d. Henricus de Hassia: Secreta sacerdotum. P. 1513. [1375.] £3 3s.

\section{Augsburg.}

Ganther Zainer. 1474 Plenarium: Evangelia cum epist. ? P. [1699.] 670 .

Gunther Zainer. n.d. Imitatio Christi. P. 1566. [4531.] \&85. [4185.] 690 .

Ganther Zainer. n.d. Schwabenspiegel. P. 1585. [1652.] £18. SS. Ulrich and Afra n.d. Historia Friderici imperatoris magni. P. 1633. [1349.] £3 5s.

Sorg. n.d. Nider: Formicarius. P. 1696. [1686.] f3 5s.

Hohenwang. 1477. Henr. de Segusio, Hostiensis: Summa super titulis decretalium. P. 1738. [1374] £3 I5s. 
N'RNBERG.

Koberger. 1477. Biblia Latina. P. 1980. [3878.] $£^{6} 5$ s.

Koberger. 1478. Antoninus: Summae pars prima. P. 1988. [1528.] 64 .

Koberger. 1479. Biblia Latina. P. 1993. [1345.] f10 5s.

Koberger. 1483. Biblia Germanica. P. 2028. [1800.] £37.

Koberger. 1493. Schedel. Liber chronicarum. P. 2084. [4028.] not returnable, $f_{6} 15$ s. [2846.] f24 ros. [1836.] f39.

Koberger. 1500. Brigitta: Revelationes. P. 2124 [1820.] £15. Creusner. n.d. Psalterium. ? P. 21 80. [1935.] $f_{1} 3$ ros.

Stuchs. 1484. Missale Romanum. P. 2259. [2894.] $\ell^{6}$.

\section{SPEIRR.}

Drach. 1490. Caracciolus: Sermones de laudibus sanctorum. P. 2380. [2405.] $\frac{1}{3} 3^{6 s}$ s.

Hist (?). n.d. Ulr. Molitoris: De laniis et phitonicis mulieribus. PP. +2443. [1905.] £5 10s.

Esslingra.

Fyner. n.d. Nider: Sermones. P. 2481. [1685.] $£^{2} 3$ s. Fyner (?). n.d. Jacobus de Theramo: Belial. germ. Not in P. [1408.] $\ell^{2} 2 s$.

LÜBECK.

Brandiss. 1475. Rudimentum novitionum. P. 2610. [1942.] $£ 3^{8}$.

Blaubruren.

Mancz. n.d. Henricus de Gorinchem: De superstitiosis casibus. P. 2654. [1368.] £3 10s.

Mrmaingen.

Kunne. n.d. Huss: Gesta Christi. P. 2817. [4195.] $£^{2}$ I8s.

UNKNOWN PLACB.

n.d. Lotharius: De miseria conditionis humanac. P. 3243. [1382.] fir Ios. 


\section{ITALY.}

Roma.

Sweynheym and Pannartz. 1470. Lactantius: Opera. P. †33'3. [1884] £29.

Sweynheym and Pannartz. 1472. Livius: Decades. P. 3326. [1385.] $£ 45 s$.

Lauer. 1470 . Johannes Chrysostomus: Homiliae super Johannem. P. 3402. [1356.] f3.

Lauer. n.d. Vergerius: De ingenuis moribus, etc. P. 3422. [1848.] \& \&19 10s.

Pannartz. 1475. Laur. Valla: Elegantiae. P. 3527. [1596.] \&8. Apud S. Marcum. 1475. Gregorius: Moralia in Job. P. $353^{6}$. [1619.] 6.5:

Herolt and Riessinger. n.d. Phil. de Barberiis: Opuscula. P. 3954- [1796.] E9.

\section{Vanezia.}

Wendelin, of Speier. 1471. Cyprianus: Epistulae P. 4032. [4471.] f.5 ros.

Wendelin, of Speier. 1472. Boccaccio: Genealogiae deorum. P. 4045. [4461.] 675 s.

Jenson. 147 I. Cicero: Epistulae ad familiares. P. 4069. [1247.] E5.

Jenson. 1471. Laur. Valla: Elegantiae. P.4071. Painted initials. [4516.] 630 .

Jenson. 1476. Plinius: Naturale historia, tradotta da Landino. P. 4099. [2699.] £2 19 (bad condition).

Jenson. 1478. Plutarchus: Vitae. P. 4113 . [1700.] $f^{6} 6 s$.

Valdarfer. (b. 9. Nov. 1471.) Dion Chrysostomus: De regno. P. 4136. $[1848.] \neq f_{19}$ Ios.

Renner. 1478. Pomponius Mela: Cosmographia. P. 4174. [1389.] $£ 3$ ros.

Bartolommea, of Cremona. 1473. Nicolaus de Ausmo: Supplementum. P. 4226. [1532.] £37 (on vellum).

Filippo, di Pietro. n.d. Georgius Trapezuntius: Commentarius in Ciceronis Philippica. P. 4263. [4515.] £3 18 s.

Filippo, di Pietro. I481. Plinius: Historia naturalis. Ital. Not in P.; H.C. 13106 . [1928.] $£ 35$ s.

Johann of Köln and Johann Manthen. 1479. Clemens V.: Constitutiones. Not in P.; H.C. "5424. [685.] £3. 
Ratdolt, with Maler and Löslein. 1478. Pomponius Mela: Cosmographia. P. 4373. [1931.] EI4 ros.

Ratdolt. I 482. Alchabitius: Liber isagogicus. P. 4382. [239.] 628 s.

Ratdolt. 1482. Euclidis elementa. P. 4383. [2536.] f14 15 s.

Ratdolt. 1485. Publicius: Artis oratoriae epitoma. P. 4399. [4538.] f6. [1398.] E5 55.

Ratdolt 1485. Abraham A ben Exra: De nativitatibus. P. 4407. [5541.] 2.12s.

Juvenis Guerinus. I477. Lucanus: Pharsalia P. 4426. [1891.] E3I5s.

Dominicus Siliprandus. n.d. Plutarchi problemata. P. 4454 [1397.] $\ell^{2}$ ios.

Luca, di Domenico. 1481. Quintilianus: Declamationes. P. 4491. [1937.] $\ell^{2}$ 12s.

J. and G. de Gregoriis. 1495. Joh. de Ketham: Fasciculus medicinae. P. 4550. [5566.] 205 s.

OEt. Scotus. I483. Blondus: Decades. P. 4575. [1546.] £5 12s. $6 d$.

Stagninus. 1499. Joh, de Ketham: Fasciculus medicinae. Not in Proctor or Burger. [4494.] I $^{6} 15^{s}$.

Pasquale and Bertochus. 1485 . S. Hieronymi vita et mors. P.4850. [1871.] $£ 3$ ros.

Paequale. I 493 . Diogenes Laertius: Vitae et sententiae philosophorum. Not in P.; H. 6203. [1927.] \& $\$ 30$.

Benalius, with Capcasa. 1491. Dante: La divina Commedia. P. 4877. [1360.] £3 6s.

Rizus, 1490. Jac. Phil. Bergomensis: Supplementum. P. 4954. [1879.] £3 ros.

Capcasa. 1493. Joh. Bapt. Cantalycius: Epigrammata. P. 4993. [4152.] $677^{5}$.

Joh. Rubeus, for L. A. Giunta 1493. Livius: Decades Ital. P. 5133. [1655.] £13. [4496.] E6.

Pincius I 495 . Missale Romanum. Not in P.; H. 11408. [1391.] 2 16s.

Petrus de Qúarengiis. 1497. Dante: La divina Commedia. P. 5482. [2406.] f14 15 s.

Aldus Manutius. 1494-95. Constant. Lascaris: Erotemata, etc. P. 5546. [1885.] £38s.

Aldus Manutius. 1495-98. Aristotle: Opera. P. 5547, 5553, $5555,5556,5565$. [2520.] E5 5s.

Aldus Manutius. I498. Politianus: Opera. P. 5567. [1930.] $£ 4$ 8s. [1701.] $£^{2}$ ros.

I2. VI. 
Aldus Manutius. 1499. Astronomici veteres. P. 5570. [1896.] f2 ros. (fragment).

Aldus Manutius. 1499. Franciscus Colonna: Poliphili hypnerotomachia. P. $5574\left[183^{8 .}\right.$ ¿90. [3062.] $£^{81}$.

\section{Fuliono.}

Neumeister. 1470. Leon. Aretinus: De bello italico. P. 5721. [1787.] £25 10s.

\section{Frrrara.}

Rossi. 1497. Jac. Phil. Bergomensis: De claris mulieribus. P. 5762. [4476.] £46.

\section{Milano.}

Valdarfer. 1474. Ambrosius: De officiis et opuscula. P. 5874. [1337.] f3 14 s.

Pachel and Scinzenzeler. 1481. Celsus: De medicina. P. 5940. [5556.] £3 12s.

\section{Firenze.}

Nicolaus Laurentii. 1478. Celsus: De medicina. P. 6116. [3788.] 69 .

Nicolaus Laurentii. n.d. Berlinghieri: Geographia. P. 6121. [1799.] £29 ros. (imp.).

Miscomini. 1492. Plotinus: Opera. P.6156. [1929.] fil 8s. Libri. 1494. Cavalca: Pungi lingua P. 6200 . 2400.] 55 s.

Libri. 1495. Savonarola: Predica e revelazioni. P. 6203. [211.] E5 2s. $6 d$.

Librí. n.d. Libro che tracta di mercatantie. [For P. Pacini.] P. 6255. [4808.] E7 5s.

Libri. n.d. Savonarola: Trattato dell' umilta. P. 6294. [1302.] fig 10s.

Buonaccorsi. 1490. Gizcopone da Todi: Laude. P. 6310. [5884.] E19 ros.

Buonaccorsi. I495. Savonarola: Compendio di revelazioni. P. 63I4. [4509.] E. 8s.

Morgiani \& Johann Petri. 1497. Franciscus de Assisis: Fioretti. Not in P. H. 7330. [1605.] 640 .

Treviso.

Bernhard, of Koln. 1478. Seneca: Opera. P.6484 [4511.] 64 .

Boloona.

Azzoguidi. 1480. Ovidius: Opera. P. 6523. [1917.] Fragmont $\{3$. 
Franciscus de Benediatus 1493. Herodianus: Historiae. P.6598. [1870.] f3.

Benedietus Hectoris. 1497.' Joh. Fr. Picus: De morte Christi : etc. P. 6634. [2608.] £3 15 s.

\section{Napour.}

Riessinger. n.d. Phalaris: Epistulae. Not in P. H. C. 12884. [1925.] $\ell^{8}$.

Moravus. 1479. Caracciolus: Quadragesimale de paenitentia. Not in P. H. 4435. [1350.] £3 35.

Unknown printer.2 n.d. Apocalypsis cum glossis Nic. de Lyra. P. 6749. [3515.] Eil 15 s.

Unknown printer. 1476. Caracciolus: Quadragesimale de peccatis. Ital. Not in P. H. 4445. [4468.] E16s.

\section{Savigliano.}

Christophorus Beyamus \& Hans Glim. n.d. Guido de Monte Rocherii : Manipulus Curatorum. Not in P. H. 8170 . [1 394.] $£ 3$.

\section{PADORA.}

Barth, de Valdezoccho. 1474. Omnibonus Leonicenus: De octo partibus orationis. P. 6762. [1886.] $£^{2} 2 s$.

\section{Mantova.}

Burster (?). n.d. Aristotelis ethica. Not in P. H. 1744 ? [1789.] \&5.

Schall. 1479. Eusebius: Historia ecclesiastica. P. 6908. [1851.] EI 17 s.

\section{VERONA.}

Johan, of Verona. 1472. Valturius: De re militari. P. 6912. [Painted initials.] [4517.] $\notin_{160 .}$

Boninus de Boninis. 1483. Valturius: De re militari. P. 6921. [1957.] £3 3.

\section{Vicenza.}

Lichtenstein. n.d. Orosius: Historiae. P. 7 144. [1915.] $\ell_{4} 5$ s. Lichtenstein. 1479. Clericus: Comment. in epistulas Ciceronis. P. 7156. [1358.] £2.

1 Dated 1487 in Catalogue. Probably a misprint, and this edition.

2 The Cataloguer in saying that Protor attributes this to the press of U. Han at Roma has been misled by a note to another book. 
Lichtenstein. 1480. Ovidius: Operz. P. 7157. [1692.] $£^{2} 18$ s. Rigo di Ca Zeno. 1499. Martianus Capella: Opera. P. 7 I 74. [933.] $£ 3$.

Bevilaqua 1487. Boccaccio: Genealogiac deorum. P. 7179. [1806.] $€ 1$.

\section{Unkmown Place.}

n.d. Nicolaus de Lyra: Quaestiones disputatae contra Judaeos. P. 7393. [1 381.] $t £^{18} 5$ s.

n.d. Martialis: Epigrammata. P. 7405. [1388.] $£^{1} 16 s$.

\section{SWITZERLAND.}

\section{Basel.}

Johann, of Amorbach. 1481. Nider: Praeceptorium. P. 756r. [1684.] \& $£ 33$ s.

Johann, of Amorbach. 1481. Vincentius Bellovacensis: Opuscula. P. 7562. [4201.] f2 15 s.

Furter. 1498. Methodius: Revelationes. P. 7738. [r69.] fi ros. Froben. 1491. Biblia latina. P. 7755. [1346.] Imp. fr.

Bergmann. 1494. Verardus: Bethicae et Granatae obsidio, victoria et triumphus. P. 7770 . [1757.] $£ 46$.

Bergmann. 1498. Brant: Varia carmina. P. 7779. [1817.] 86155.

\section{FRANCE.}

Paris.

Gering, Kranz \& Friburger. 1475. Gregorius: Homiliae. P. 7844. [1369.] fi5.

Levet. 1486. Coutoumier d'Anjou et de Maine. P. 8053. [2104] 69 iss.

Higman \& Hopgl. 1497. Aristoteles: Decem librorum moralium tres conversiones. Not in P. H. C. 1761. [1339.] f1 10s.

Le Caron. n.b. 1488. Caesar: De bello gallico. Gall. [For A. Verard.] P. 8141. [1821.] £31.

Bocard. 1497[98]. Gaguinus: De origine et gestis francorum. Not in P. H. C. 7411. [1367.] f28s.

Pigouchet. 1499. Flores sancti Bernardi. P. 8198 ? [1542.] £3 I os. 1499. Heures i l'usage de Rome [For S. Vostre.] Not in P. [4484] Sold as imperfea. E4 17s. 6d. 
Verard. n.d. Horae secundum usum romanae curiae. P. 8420 . [4483.] Imp. fir 17 ros.

Verard. n.d. Froissart: Chroniques. P. 8452. [1365.] Vol. II. only. £5 15 s.

Verard. n.d. Quintus Curtius: Historia Alexandri magni. Gall. Not in P. H. 5887. [1844.] £27.

\section{LYON.}

Huss. 7 April. 1487[88]. Bartholomaeus Anglicus: Le proprietaire des choses. Not in P. H. 2516. [1865.] \&38.

Dupre. 1491. La mer des histoires. 2 vol. Not in P. Cop. II. 3992. [1902.] $£^{6} 5$.

Maillet. 149]. Le songe du vergier. P. +8622 . [1946] Erg. Sacon. 1498. Brant: Stultifera navis. P. 8671. [1816.] £7 ros.

\section{AbBeville.}

Gtrard. 1486. Augustinus: La cité de Dieu. P. 8763. [1793.] [Vol. I. only.] Eib.

\section{Rouser.}

Le Talleur. n.d. Nic. Statham: Abridgment. [For Pynson.] P. 8768. [1407.] Ł14 10s. [4075.] £40.

Angourime.

Alanus \& Calvinus. n.d. Eruditorum paenitentiale. P. 8793. [1850.] $£^{20}$.

\section{HOLLAND.}

\section{DrLFt.}

Van der Meer \& Yemantszoen. 1484 Jacobus de Voragine: Passionael. Winterstuc Not in P. CA. 1760. [146.] \&4

\section{DeventrR.}

Pafraet. 1491. Exhortationes novitiorum: otc. P. 8998. [1363.] 62.

Z wolle.

Van Os 1490. Hieronymus: Vaderboeck. P. 9135 . [154] 6555. Van Os. 1495. Bernardus: Sermones in duytessche. P. 9145. [125.] \&8. 


\section{BELGIUM.}

\section{Louvain.}

Johann, of Paderborn. n.d. Platina: De honestate voluptate et valitudine. P. 9283. [2550.] £3.

Anvirs.

Bac. (a. 19 Aug. 1493.) Exsequiae dom. Frederici III. imp. Not in P. Cop. II. 2584. [1857.] EI 12 s.

\section{ENGLAND.}

\section{WESTMINSTER.}

Caxton. [b. 1479.] Boethius: Of the consolation of philosophy. P. 9630. [1352.] 2 leaves. \& fig.

Caxton. 2. 8. Mar. 148f. The mirror of the world. P. 9638 . [1827.] 36 leaves. $f_{0} 103$.

Cuxton. 1481. The boke of Tulle of old age, atc. P. 9640. [5497.] 'Of friendship' only. f125.

Caxton. [2. 2 July 1482.] Higden: Polychronicon. P. 9645. [1351.] II leaves, $£ 43$.

Caxton. 2. 20. Nov. 1483 . Jac. de Voragine: The golden legend. P. 9655. [1828.] Imp. 185 .

Caxton. n.d. Lydgate: Life of our lady. P. 9665-6. [1352.] 2 leaves. 4 fig.

Caxton. n.d. The royal book. P. 9671. [4256.] Leaves in facs., etc. $£ 295$.

Wynkyn de Worde. I495. Vitas patrum. angl. P. 9697. [4257.] fisI.

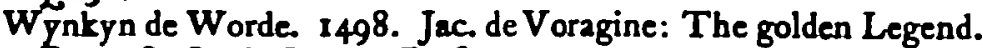
P. 9708. [2564] 220 ff. £.10 5s.

\section{LONDON.}

Machlinia. n.d. Nova statuta. P. 9765 . [2370.] £223.

Machlinia n.d. Statuta anni i Rich. III. Now P. 97652. [2369.] fragment, $E_{4}$.

Pynson. 1493. Dives et pauper. P. 9782. [4258.] E75; [3530.] 3 ff. in facs. $41^{1}$; [5836.] Imp. E15. 


\section{PORTUGAL.}

\section{Lajria.}

Abraham ben Samuel Dortas. 1496. Zacuthus: Almanach perpetuum. Not in P. Haebler, 720. [6254-] £17.

\section{UNIDENTIFIED.}

n.d. Accursius: Index locorum in Comment Cresur. Brunet I. 34. [1549.] \&3 ros.

Aesopus. Fabulae. [1783.] fi7 5s.

n.d. Aesopus graecus. [1782.] $€ 3$ 18s.

n.d. Basilius: Opuscula, atc. H. 2684. [1848.] 6 . 19 10s.

n.d. Guido de Monte Rocherii: Menipulus curatorum. H.`8162. [1684.] Z $\{33$ s.

n.d. Jac. de Voragine: Legenda aurea. [1964.] £45.

n.d. Jac. de Voragine: Sermones aurei de sanctis. [14II.] E5 25. 6d.

n.d. Ludolphus de Saxonia: Le grand vita Christi en francais. [Part I-2 only.] [1893.] f5 55.

n.d. Pragmatica sanctio Caroli VII. [1 381.$]+6^{18} 5 \mathrm{~s}$.

1482. Breviarium Romanum. H. 3904. [2839.] E18 IOS.

R. A. Peddie. 